\title{
Flow rate estimation in large depth-of-field micro-PIV
}

\author{
A. Kloosterman $\cdot$ C. Poelma $\cdot$ J. Westerweel
}

Received: 31 May 2010/Revised: 21 October 2010/Accepted: 17 November 2010/Published online: 12 December 2010

(C) The Author(s) 2010. This article is published with open access at Springerlink.com

\begin{abstract}
In micro-Particle Image Velocimetry, the requirement of a large field-of-view often results in a large depth-of-correlation, i.e. large depth of the measurement volume. When the velocity varies substantially over the depth-of-correlation, special attention should be paid to a correct interpretation of the measured velocities. When a specialized microscope is needed to meet the requirements of a setup, the resulting more complex optical arrangements can have additional effects on the measurement results. In order to determine flow parameters such as the flow rate, it is sufficient to have a robust estimate of the maximum velocity when the flow is Poiseuille flow. In this paper, an interpretation of the results from particle image velocimetry measurements with low magnification in a round capillary is given for two types of microscopes: a conventional and a specialized microscope. The measured velocity appears to be lower than the maximum velocity, yet is still above the average velocity. The interpretation of the measured velocity differs for the two types of microscopes. The under-estimation of the maximum velocity obtained from the conventional microscope remains small (within 6\%) for low-magnification measurements, while the under-estimation of the maximum velocity obtained from the specialized microscope increases up to $25 \%$ for a large depth-of-correlation. The images of the in- and outof-focus particles turn out to play a crucial role in this difference between the two microscopes. Validation of the optical properties of a microscope is important, especially
\end{abstract}

\footnotetext{
A. Kloosterman $(\bowtie) \cdot$ C. Poelma $\cdot$ J. Westerweel Laboratory for Aero and Hydrodynamics, Faculty of Mechanical, Maritime and Materials Engineering, Process and Energy department, Delft University of Technology, Mekelweg 2, 2628 CD Delft, The Netherlands e-mail: a.kloosterman@tudelft.nl
}

for specialized microscopes where particle images deviate substantially from the existing theory, and this theory is also used to derive the analytical expression for the depthof-correlation. A procedure is recommended to obtain a correct interpretation of the measured velocity. This procedure is generally applicable, but mainly of importance for specialized microscopes.

\section{Introduction}

In micro-Particle Image Velocimetry (micro-PIV) the depth of the measurement volume is determined by the depth-of-correlation (DOC). The DOC is inversely proportional to the numerical aperture (NA) of the microscope objective; the larger the NA, the smaller the DOC. In general, objectives with a large magnification also have a higher NA. This implies for many practical situations where a small DOC is used (i.e., with a high NA and high magnification), that the field-of-view is very small. This can be a severe limitation in microfluidic applications where a large field of view is desirable, for example in complex microfluidic systems or in biological applications (e.g., the investigation of a blood vessel network). As the flow is often Poiseuille-like, a robust estimate of only the mean or maximum velocity can provide parameters such as the flow rate and wall shear stress, which are in many flows more important than the exact shape of the profile. Also, certain applications require specialized microscopes. For example, the investigation of microscale biological flows require upright microscopes, or microscopes with a long working distance, or even microscopes that can be used with combinations of different optical configurations (e.g., the combined stereoscopic/upright single-view microscope used by Vennemann et al. 2006). The optical properties of 
various microscopes can be quite different, which means that microscopes with more complex optical arrangements, such as microscopes with a zooming capability, can be quite different from conventional microscopes. In this paper, we investigate the effect of the DOC on the measured flow velocity as a function of the DOC relative to the typical dimension of the flow. In other words, how should the measured displacement be interpreted when the velocity varies substantially over the DOC. It appears that the measured velocity cannot be considered as a simple volume average of the velocity over the measurement domain. This also depends on the optical characteristics of the microscope, and in particular on the way in which the particle image diameter varies with the distance from the (nominal) focal plane.

\subsection{Depth-of-correlation}

The DOC describes the depth over which particles contribute significantly to the spatial correlation (Olsen and Adrian 2000). When the DOC increases, also particles outside the focal plane contribute to the correlation function, which influences the measured velocity. The DOC is thus an estimate of the thickness of the measurement volume. It can be calculated by (Olsen and Adrian 2000):

$\mathrm{DOC}=2\left[\frac{1-\sqrt{\varepsilon}}{\sqrt{\varepsilon}}\left(f^{\# 2} d_{\mathrm{p}}^{2}+\frac{5.95\left(M_{0}+1\right)^{2} \lambda^{2} f^{\# 4}}{M_{0}^{2}}\right)\right]^{1 / 2}$

where $f^{\#}$ is the aperture number of the lens, $d_{\mathrm{p}}$ the particle diameter, $M_{0}$ the nominal magnification, $\lambda$ the wavelength of light emitted by the particle, and $\varepsilon$ a threshold value for the intensity of particle images that contribute to the measured spatial correlation (typically $\varepsilon$ is chosen to be $0.01)$.

The DOC is mainly sensitive for changes in the particle diameter $d_{\mathrm{p}}$ and the aperture number $f^{\#}$. In the studies described in Vennemann et al. (2006) and Poelma et al. (2008, 2010), artificial (bio-inert) tracer particles were added to the blood to obtain velocity profiles from PIV measurements. PIV measurements are also possible without these artificial tracer particles, by using the red blood cells (RBCs) as tracer particles (Lee et al. 2007; Sugii et al. 2002). For typical experimental values, e.g. $f^{\#}=1.0$, $M_{0}=20, \lambda=590 \mathrm{~nm}$, and $\varepsilon=0.01$, the DOC increases from 11 to $43 \mu \mathrm{m}$ when RBCs with a diameter of $7 \mu \mathrm{m}$ are used instead of particles with a diameter of $1 \mu \mathrm{m}$. While the use of RBCs has the advantage that the system is not affected, it also results in a fourfold larger DOC.

Some applications demand large fields-of-view, i.e. low microscope magnification (e.g. studying a blood vessel network). With most microscopes, a reduction in the magnification results in a lower numerical aperture and thus a higher aperture number $f^{\#}$, and eventually to a larger DOC. As a result, using low magnifications will not only influence the resolution and averaging characteristics in the directions of the measurement plane, but also in the direction normal to the plane. Taking the same values for the parameters in the formula of the DOC as above, the DOC will increase from 11 to $40 \mu \mathrm{m}$, when changing the magnification from the previous value of $20 \times$ to $10 \times$, with a corresponding aperture number $f^{\#}=2.0$.

In Fouras et al. (2007), the averaging problem due to volume illumination is treated for particles with constant contribution in the out-of-plane direction. Earlier work from e.g. Bourdon et al. (2004a) and Rossi et al. (2010) describe methods for modifying the effect on the measured velocity due to the depth-of-correlation by applying image preprocessing techniques. Fouras et al. (2009) suggest to use decomposition of the correlation function in order to obtain the actual velocity data. Another procedure for decreasing the measurement depth is using selective seeding. This is described in Mielnik and Saetran (2006). This may be a solution for certain situations, but for in vivo measurements, this method seems impractical. In this study, the focus will be on interpreting the measured velocity correctly.

The expression for the DOC in (1) is based on certain assumptions. For example, the size and intensity distribution of a particle image are modeled for both in- and outof-focus particles under ideal conditions (e.g. a single thin lens and equally distributed light intensity). The particle image diameter $d_{\tau}$ as a function of $z$, where $z$ is the distance to the object plane, is modeled as (Olsen and Adrian 2000; Bourdon et al. 2004b):

$d_{\tau}^{2}(z)=M_{0}^{2} d_{\mathrm{p}}^{2}+5.95\left(M_{0}+1\right)^{2} \lambda^{2} f^{\# 2}+\frac{M_{0}^{2} z^{2} D_{\mathrm{a}}^{2}}{\left[\left(n_{f} / n_{l}\right)\left(s_{0}+z\right)\right]^{2}}$

where $D_{\mathrm{a}}$ is the lens aperture diameter, $s_{0}$ the distance from the lens to the object plane, $n_{f}$ the refractive index of the fluid containing the particles, and $n_{l}$ the refractive index of the lens immersion medium. The first two terms in expression (2) represent the particle image diameter for a particle located in the object plane. This is a combination of the geometric image (first term) and the diffraction (second term). The last term describes the geometric spreading of the particle image when a particle is not located in the object plane, but at a distance $z$ to the object plane. The particle intensity distribution $I(r, z)$ is assumed to be Gaussian and is modeled as (Olsen and Adrian 2000):

$I(r, z)=\frac{J_{\mathrm{p}} D_{\mathrm{a}}^{2} \beta^{2}}{4 \pi d_{\tau}^{2}\left(s_{0}+z\right)^{2}} \exp \left(\frac{-4 \beta^{2} r^{2}}{d_{\tau}^{2}}\right)$ 


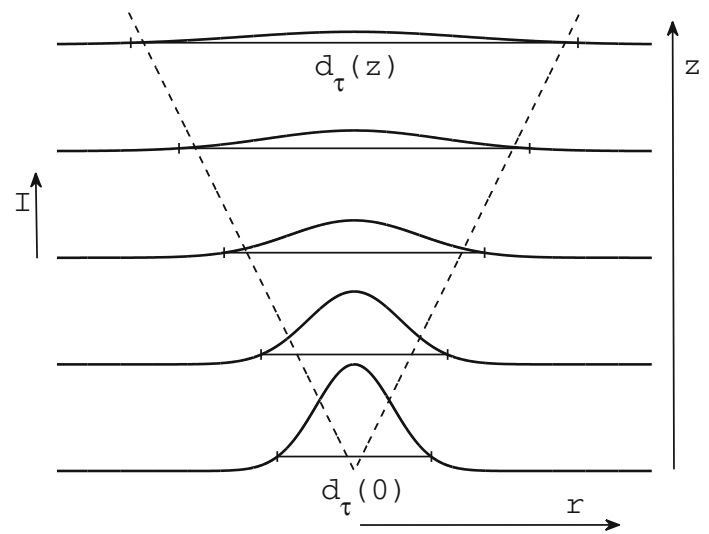

Fig. 1 Schematic representation of the cross sections of particle images for different $z$-positions based on expressions (2)-(3). The particle image diameter, $d_{\tau}(z)$, increases for increasing $z$, while the peak intensity $I(0, z)$ decreases. The dashed line represents the geometrical spreading of an image of an out-of-focus particle (last term in expression (2))

where $r$ is the distance to the particle center, $J_{\mathrm{p}}$ the flux of light emitted from the particle surface, and $\beta$ a constant that is often taken as $\beta^{2}=3.67$ (Adrian and Yao 1985). For this value of $\beta^{2}$, the Gaussian distribution is the best approximation for the Airy distribution. This model appears to be in good agreement with experimental data (Olsen and Adrian 2000). In Fig. 1, the $z$-dependency of a particle image is illustrated.

Micro-PIV measurements in for example biological setups may require specialized microscopes. An upright microscope with one or more additional features, such as a long working distance, stereoscopic imaging abilities, and a zoom function may be desirable. The first part of this study consists of the verification of the assumptions for the in- and out-of-focus particle images for two types of microscopes: the particle image diameter as a function of $z$ and the intensity distribution are determined experimentally and are compared with the expressions in (2)-(3). The two types of microscopes are an inverted epifluorescent microscope in combination with different interchangeable objectives to obtain different magnifications and an upright epifluorescent microscope in combination with one objective with long working distance and a zoom function to obtain different magnifications in a specific range. Due to the differences in optics used in the microscopes, resulting from the different features, the way particles are imaged may be affected. This, in turn, influences the DOC, and the measured velocity can therefore deviate from the velocity based on the calculated DOC by (1). A relatively simple objective (i.e. one fixed magnification) is already very complex, with many more optical elements than the single thin lens that is used to model it. ${ }^{1}$

\footnotetext{
${ }^{1}$ For examples of objective schematics, we refer to e.g. the website of Zeiss, http://www.zeiss.de.
}

To gain a better insight into how measured velocities relate to the actual velocity in the presence of local gradients, the accuracy of the micro-PIV method will be investigated experimentally for two commonly used types of microscopes. In the second part of this study, an in vitro experiment is performed in a known reference flow: a glass capillary is used to model a blood vessel. With a micro-PIV system, measurements are performed for different magnifications. The measured maximum velocities are compared with the known maximum velocities to investigate the influence of the spatialaveraging characteristics, given the in-plane and out-ofplane (DOC) spatial resolution. The experimentally measured data for $d_{\tau}(z)$ is used to provide a better understanding of the measured velocity.

In applications that require a large field-of-view (i.e. low image magnification), such as application of micro-PIV to biological flows, it is difficult or sometimes even unfeasible to have a sufficiently small DOC; note that in most studies, it is implicitly assumed that the PIV measurement plane represents a thin slice coinciding with the center-plane of a blood vessel. When the DOC becomes larger than the diameter of the blood vessel, the measured velocity is often interpreted as an average of the velocity due to the depth averaging.

\section{Methods}

\subsection{Setup}

For our investigation, we considered two microscopes: a conventional inverted epifluorescent microscope in combination with different interchangeable objectives to obtain different magnifications (Zeiss, Axiovert 200) and an upright epifluorescent microscope with zoom function in combination with a single objective to obtain different magnifications (Leica MZ 16 FA) (see Fig. 2 for pictures of both microscopes). With the inverted microscope, three different objectives are used, with magnifications $M_{0}=$ $20 \times, 10 \times$, and $5 \times$ (with numerical apertures NA $=0.5$ (Zeiss Plan Neofluar), 0.3 (Zeiss Plan Neofluar) and 0.25 (Zeiss Fluar), respectively); see Table 1. In order to traverse the focal plane, a piezo objective positioner (Piezo Jena) is combined with the inverted microscope. The upright combi-microscope can be used for stereoscopic and normal viewing. The microscope is also equipped with a long working distance. These additional features can be useful or are sometimes even necessary for application of micro-PIV to biological flows, e.g. in vivo measurements in a chicken embryo (Vennemann et al. 2006; Poelma et al. 2008, 2010). The upright combi-microscope has a zoom functionality and is used together with a single objective 
Fig. 2 Pictures of the two microscope used in this study: a the inverted microscope from Zeiss (Axiovert 200) and b the upright combi-microscope from Leica (Leica MZ 16 FA)
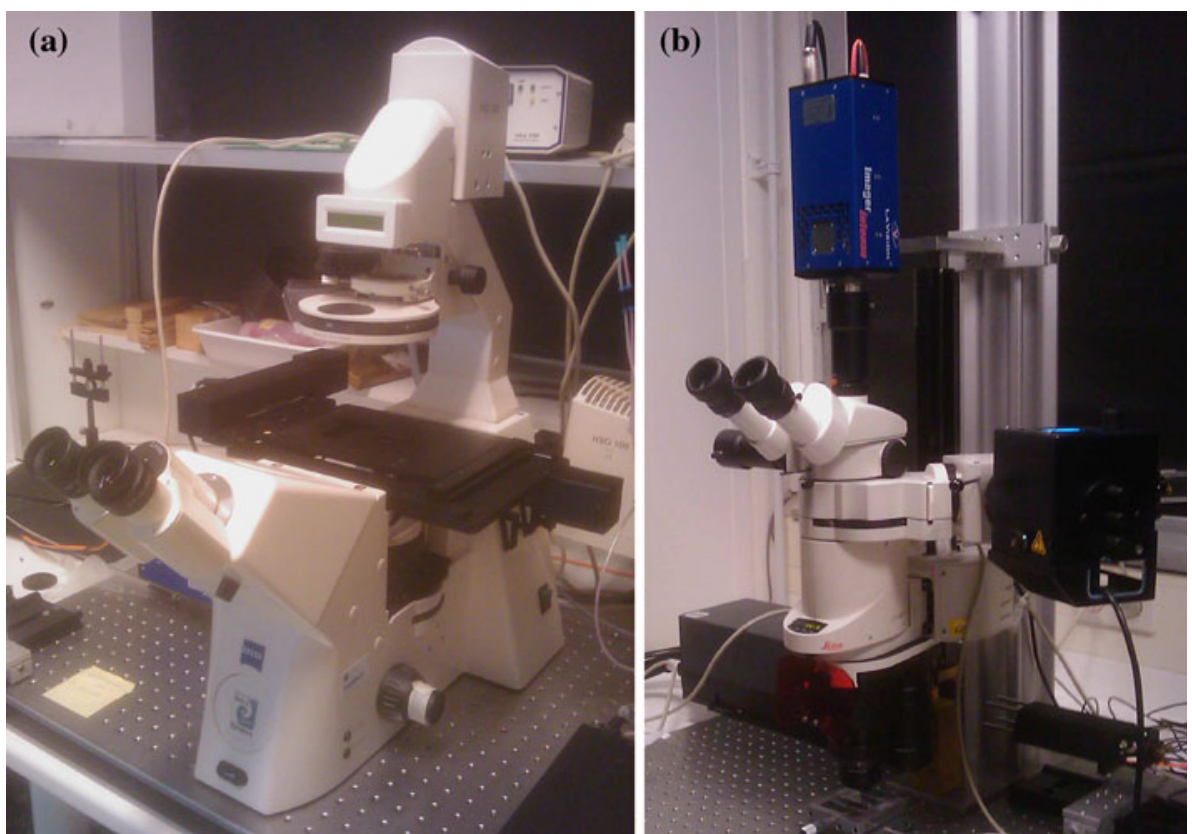

Table 1 Magnifications and corresponding numerical apertures, particle image diameters, depths of correlation, exposure time delays $\Delta t$, and sizes of the interrogation windows (IW) for both microscopes $\left(\lambda=590 \mathrm{~nm}, d_{\mathrm{p}}=0.56 \mu \mathrm{m}\right.$ and $d_{\mathrm{p}}=1.28 \mu \mathrm{m}$ and $\left.\varepsilon=0.01\right)$

\begin{tabular}{llllrl}
\hline \multicolumn{6}{l}{ Inverted microscope } \\
\hline$M_{0}$ & NA & $d_{\tau}(\mathrm{px})$ & DOC $(\mu \mathrm{m})$ & $\Delta t(\mu \mathrm{s})$ & IW $(\mathrm{px})$ \\
\hline 20 & 0.50 & 4 & 10 & 375 & $40 \times 64$ \\
10 & 0.30 & 3 & 27 & 750 & $20 \times 64$ \\
5 & 0.25 & 2 & 42 & 1,500 & $10 \times 64$ \\
\hline
\end{tabular}

Upright combi-microscope

\begin{tabular}{|c|c|c|c|c|c|c|c|}
\hline \multirow[t]{2}{*}{$M_{0}$} & \multirow[t]{2}{*}{ NA } & \multicolumn{2}{|c|}{$d_{\tau}(\mathrm{px})$} & \multicolumn{2}{|c|}{$\mathrm{DOC}(\mu \mathrm{m})$} & \multirow[t]{2}{*}{$\Delta t(\mu \mathrm{s})$} & \multirow[t]{2}{*}{ IW (px) } \\
\hline & & 0.56 & 1.28 & 0.56 & 1.28 & & \\
\hline 38 & 0.50 & 6 & 8 & 7 & 9 & 200 & $76 \times 64$ \\
\hline 30 & 0.48 & 5 & 6 & 8 & 10 & 250 & $60 \times 64$ \\
\hline 25 & 0.45 & 4 & 6 & 9 & 12 & 300 & $50 \times 64$ \\
\hline 20 & 0.40 & 4 & 5 & 13 & 15 & 375 & $40 \times 64$ \\
\hline 15 & 0.33 & 4 & 4 & 19 & 22 & 500 & $30 \times 64$ \\
\hline 10 & 0.24 & 3 & 4 & 39 & 42 & 750 & $20 \times 64$ \\
\hline 8 & 0.19 & 3 & 3 & 66 & 68 & 1,000 & $16 \times 64$ \\
\hline 5 & 0.14 & 3 & 3 & 130 & 132 & 1,500 & $10 \times 64$ \\
\hline 4 & 0.11 & 3 & 3 & 224 & 226 & 2,000 & $8 \times 64$ \\
\hline 3 & 0.09 & 3 & 3 & 349 & 352 & 2,400 & $6 \times 64$ \\
\hline
\end{tabular}

The numerical apertures for the upright combi-microscope are extracted from the specifications provided by Leica

with magnification $M_{0}=5 \times$ and numerical aperture NA = 0.5 (Leica FluoCombi III). This combination provides magnifications in the range of $2.8-46 \times$ with corresponding numerical apertures in the range of $0.08-0.5$. For this microscope, moving the focal plane is accomplished by moving the built-in translation stage of the microscope, which is controlled by the recording software of LaVision DaVis. Furthermore, for both microscopes, we use a PCO Sensicam QE camera $(1,376 \times 1,040$ pixels $)$ for image recording and a diode-pumped Nd:YLF laser (New Wave Pegasus) for illumination. ${ }^{2}$

For the PIV measurement, a glass capillary with an inner diameter of $148 \mu \mathrm{m}$ (TSP150375, Polymicro Technologies) is used, which is comparable in dimension to typical dimensions of commonly used micro-channels and to the larger blood vessels of the vitelline network of a chicken embryo (Poelma et al. 2008). To allow optical access, part of the external coating is removed by treating it with sulfuric acid and heating it, not exceeding $130^{\circ} \mathrm{C}$, to preserve the glass quality. A 1-ml syringe is connected to the glass capillary, and the syringe is placed into a syringe pump (Model 101, KD Scientific). Fluid is pumped into the glass capillary at a flow rate of $5 \mu \mathrm{l} / \mathrm{min}$. The fluid contains tracer particles and glycerin to increase the refractive index to 1.45 , which closely matches the refractive index of the glass capillary. This minimizes the difference in refractive indices of the fluid and glass to reduce refraction effects that would otherwise distort the image. The section of the capillary that is observed is placed on a microscope slide and a cover glass is placed on top of it (see Fig. 3). To further reduce any effects related to differences in

\footnotetext{
${ }^{2}$ In our setups, a $1 \times$ coupling between the microscope and the camera is used. The large field-of-view can be maintained for increasing magnifications when the coupling is lowered. The effective numerical aperture is likely to increase. As a side effect, this solution might cause vignetting problems.
} 


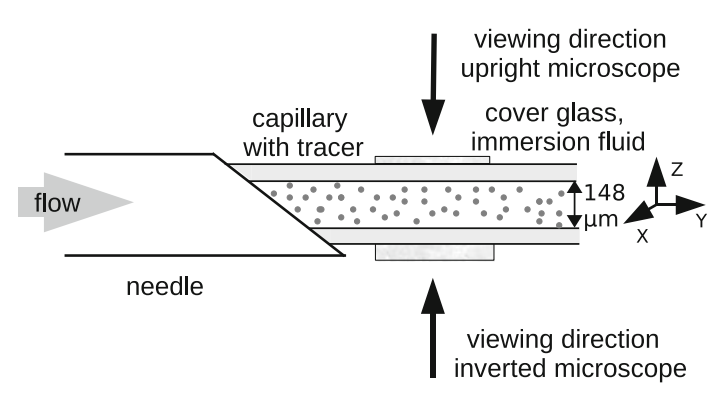

Fig. 3 Schematic representation of the setup for the PIV experiments (not to scale). The fluid, containing tracer particles and glycerin, is pumped through a glass capillary with a diameter of $148 \mu \mathrm{m}$. PIV images are recorded with a camera from below when the inverted microscope is used and from above when the upright combimicroscope is used

refractive index, the space around the glass capillary, between the microscope slide and cover glass, is filled with the same fluid as inside the capillary, i.e. glycerin with a refractive index of 1.45 .

The entrance length $L_{\mathrm{e}}$, i.e. the minimum length that is required for establishing a fully developed laminar flow, for laminar flows can be calculated by (White 2008): $L_{\mathrm{e}} / d=0.06 \mathrm{Re}$, where $d$ is the inner diameter of the capillary. The prescribed flow rate corresponds to a flow with a Reynolds number in the capillary of $10^{-2}$, so that the entrance length is very small $\left(\approx 10^{-7} \mathrm{~m}\right)$. The flow will thus be fully developed at the measurement location ( $\approx 10 \mathrm{~cm}$ downstream of the syringe).

The tracer particles are polystyrene spheres (Microparticles $\mathrm{GmbH}$ ) with a diameter of $1.28 \mu \mathrm{m}$. The tracer particles contain a fluorescent dye (Rhodamine B) and are coated with poly-ethylene-glycol (PEG), which avoids particle coagulation and also avoids that the tracer particles attach to the wall of the capillary. To check for unexpected results related to the particle size, the PIV measurements are repeated with similar particles, but with a diameter of $0.56 \mu \mathrm{m}$. These PIV measurements are only done on the upright combi-microscope. Due to the more complex optical configuration of the microscope and the larger range of magnifications that we investigated, the possible deviations are more likely to occur for this microscope.

\subsection{Particle image diameter and depth-of-correlation}

We first determined for both microscopes the particle image shape and diameter as a function of the distance $z$ from the (nominal) focal plane. We then performed a series of PIV measurements of the flow in the capillary for different measurement planes with respect to the center line (i.e., $z=0$ ) of the capillary, in order to determine the velocity profile at $z=0$. The measurements on the inverted microscope are done with different objectives with different magnification and numerical aperture. The upright combi-microscope has a zoom functionality and is used together with a single objective $\left(M_{0}=5, \mathrm{NA}=0.5\right)$. The effective numerical aperture is not a fixed value, as it depends on the effective magnification. The aperture number $f^{\#}$ is used in (5) and (1), which is related to the numerical aperture, and can be computed with (Meinhart and Wereley 2003) ${ }^{3}$

$f^{\#}=\frac{1}{2}\left[\left(\frac{n}{\mathrm{NA}}\right)^{2}-1\right]^{1 / 2}$

where $n$ is the refractive index of the immersion medium between the object and the lens ( $n=1.0$ for air).

The theoretical values for the particle image diameter in the focal plane $d_{\tau}(z=0)$ can be computed with (Adrian and Yao 1985; Adrian and Westerweel 2010):

$d_{\tau}=C \sqrt{M^{2} d_{\mathrm{p}}^{2}+5.95(M+1)^{2} \lambda^{2} f^{\# 2}}$, with $C=0.74$

The value of $C$ is defined such that the width of the particle image corresponds to the $e^{-2}$ diameter of the intensity distribution, normalized by the peak intensity. The values for $d_{\tau}$ can be found in Table 1 .

For every magnification $M_{0}$ and corresponding numerical aperture NA, the DOC is calculated using equation (1) with $\lambda=590 \mathrm{~nm}$ (this wavelength represents the range of emitted wavelengths by the fluorescent particles), $d_{\mathrm{p}}=1.28 \mu \mathrm{m}$ and $\varepsilon=0.01$. For the measurements on the upright combi-microscope, the DOC is also calculated with $d_{\mathrm{p}}=0.56 \mu \mathrm{m}$. The results are given in Table 1 and are represented graphically in Fig. 4.

\subsection{Data acquisition and analysis}

In order to determine the particle image diameters and intensity distributions, the focal plane has to be traversed with respect to tracer particles at a fixed location. To accomplish this, a set of images is taken for different $s_{0}$ positions, i.e. distances between the lens and the particle. Fluid containing tracer particles is placed between a microscope slide and a cover glass. During the measurements, there was no observable flow. The appropriate distance between subsequent parallel image planes depends on the magnification and varies between 1.5 and $2.5 \mu \mathrm{m}$ for the combination inverted microscope and electric piezo objective positioner. For the upright combi-microscope, the distance between subsequent parallel image planes varies between 5 and $12 \mu \mathrm{m}$. These variations do not depend on the magnification, but are due to mechanical limitations of the microscope and are registered and taken into account.

\footnotetext{
${ }^{3}$ Instead, the approximation $f^{\#}=1 /(2 \mathrm{NA})$ can also be used.
} 


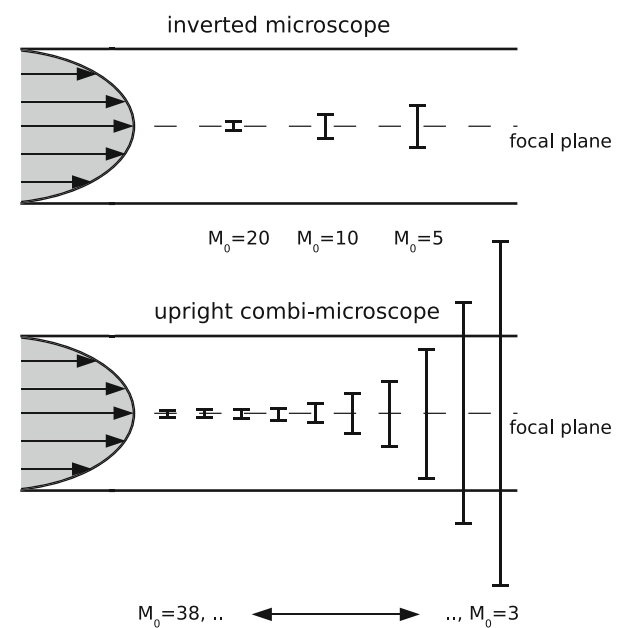

Fig. 4 The theoretical values for the depth-of-correlation (DOC) relative to the capillary diameter for different magnifications $M_{0}$ for both microscopes, given by the parameters in Table 1 . To obtain different magnifications with the inverted microscope, different objectives are used. Because of the zoom functionality of the upright combi-microscope, different magnifications can be obtained with a single objective

To reduce the signal-to-noise ratio, 200 images are recorded for every $s_{0}$ position. The average of these 200 images is used for further analysis. The image quality is improved by applying a mean filter when needed and the affected parameters are corrected for this.

The intensity distribution of a particle is assumed to be Gaussian (for particle images, see Figs. 6, 7), and the edge of the particle, defined as where the intensity has dropped to $e^{-2}$ of the peak value occurring in the center, has to be determined. This is done by fitting a second-order polynomial to the natural logarithm of the intensity. The diameter and the peak intensity of the particle can be expressed in terms of the fitting parameters. This is repeated for all planes with consecutive $z$-positions. Another way to determine the average particle image diameter is by using the auto-correlation of the total image (Adrian and Westerweel 2010). Due to the finite space between the microscope slices, particles with slightly different distances to the lens are imaged so that the particle images do not have equal diameters. As a consequence, the results might be biased toward a slightly larger particle image diameter with respect to a situation where all tracer particles are exactly in the focal plane. Therefore, the auto-correlation method may not be suitable in this study, while the current method can handle this.

The data acquisition of the PIV measurements in the capillary is done using single-exposure/double-frame recording. The flow rate is kept constant (within 1\%) during all measurements. For each series of PIV measurements corresponding to one magnification, the maximum pixel displacement is kept more or less constant at a value of approximately 10 pixels, by adjusting the time between two frames $(\Delta t)$. This implies a small $\Delta t$ for the highest magnification, and increasingly larger exposure time delays for decreasing values of $M_{0}$. In our measurements, we thus varied the values of $\Delta t$ between 200 and $2,400 \mu$ s. The exact values for each magnification can be found in Table 1 .

The maximum velocity occurs at the center of the capillary. To determine when the focal plane coincides with the center-plane of the capillary, scanning PIV is applied: multiple PIV recordings are done for different planes normal to the $z$-axis. The distance between subsequent parallel planes is $5 \mu \mathrm{m}$ for the inverted microscope. For the upright combi-microscope, the distance between subsequent parallel planes varies and is less than $12 \mu \mathrm{m}$ (the variation is due to the mechanical limitations of the microscope). The measurement plane containing the highest velocities is considered to be the center-plane of the capillary and will be used for further analysis. Assuming a parabolic flow profile, the error in the maximum velocity resulting from the inaccuracy of the $z$-position is estimated to be less than $1 \%$ for both microscopes.

In every measurement plane, 500 image pairs are recorded. For the analysis of the images, correlation averaging is applied: the local cross-correlation data is averaged to determine the displacement field (Meinhart et al. 2000). In fully developed laminar flows, only the streamwise velocity component is non-zero. Hence, in our capillary, the velocity in the $y$-direction $(v)$ dominates and $\partial v / \partial y=0$, and therefore, it is appropriate to use rectangular interrogation windows (with respect to the coordinates defined in Fig. 3). The analysis with rectangular interrogation windows is done in three passes with $50 \%$ overlap and grid refinement by a factor two for the second iteration is applied. The lengths of the interrogation windows in the direction of the $y$-axis (streamwise direction) are fixed, but to exclude the effects of the in-plane gradients, the lengths of the interrogation windows in the direction of the $x$-axis are variable and depend on the magnification. The sizes of the interrogation windows are chosen in a way such that for every magnification, approximately 24 interrogation windows are used in the $x$ direction of the plane through the capillary. For example, the sizes of the interrogation windows used for the analysis for $M_{0}=25$ for the three iterations are $100 \times 128$, $50 \times 64$, and $50 \times 64$. As a result, the final sizes of the interrogation windows vary from $76 \times 64$ for the highest magnification on the upright combi-microscope $\left(M_{0}=38\right)$ to $6 \times 64$ for the lowest magnification on the upright combi-microscope $\left(M_{0}=3\right)$, see Table 1 . The PIV evaluation is done with an in-house Matlab code, which is also used by Poelma et al. (2008, 2010).

From the PIV data, the displacement is computed by determining the peak location in the correlation function. 


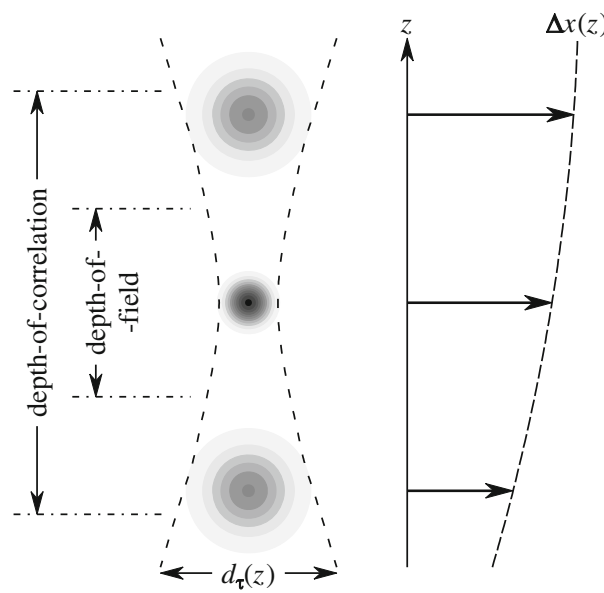

Fig. 5 The correlation function $R(s)$ is the summation of the contributions of all particles within the depth-of-correlation $(D O C)$ : $R(s)=\int R(s \mid z) f(z) \mathrm{d} z$. When the DOC increases, the influence of the particles outside the focal plane becomes larger and this may effect

This correlation function is the summation of the contribution of all particles imaged on the area corresponding with the interrogation window, i.e. the summation of the individual correlation functions resulting from all these particles. When gradients are present, the centers of the individual correlation peaks may no longer coincide, and as a result, the measured displacement may be influenced, see Fig. 5. For a better understanding, the experimental results are compared with a model: the reconstructed correlation function. In this model, the correlation function is reconstructed by summing up the individual correlation functions. This can be done here because the shape of the velocity profile is known. An individual correlation function can be obtained by computing the auto-correlation of the particle image and centering this around the corresponding displacement. Assuming the particle image to be Gaussian, the auto-correlation is also Gaussian and can be expressed in terms of the diameter and peak intensity of the particle image. The particle image diameter $d_{\tau}(z)$ and normalized peak intensity $\tilde{I}_{\text {peak }}=I(0, z) / I(0,0)$ as functions of $z$ are obtained in two different ways:

(a) The theoretical particle image diameters and normalized peak intensities computed by (2) and (3) are used (Olsen and Adrian-based reconstructed correlation function),

(b) The measured particle image diameters and normalized peak intensities from the first part of this study (Sect. 3.1) are used (experiment-based reconstructed correlation function).

In combination with the corresponding centers of the individual correlation functions, known from the parabolic

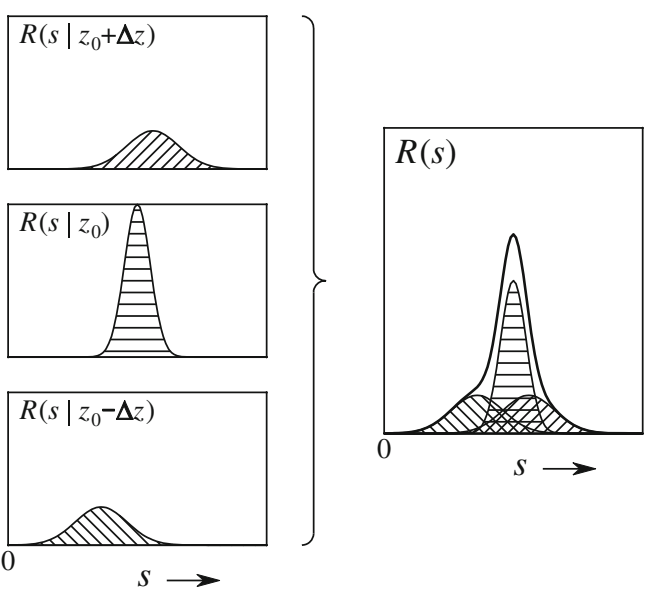

the measured displacement. Note that in this figure the maximum velocity does not coincide with the focal plane, which is the case in this study. After: Adrian and Westerweel (2010)

velocity profile, the individual contributing correlation functions are determined. This way, we can reconstruct the resultant correlation function incorporating the contributions of all particles with different $z$-positions.

\section{Results}

\subsection{Particle images}

For both microscopes, the particle image diameter and the peak intensity for different $z$-positions are determined experimentally for different magnifications, see Table 1 . The peak intensities are normalized by dividing them by the maximum peak intensity, occurring at $z=0$. For the upright combi-microscope, the diameters and peak intensities of the particle images could not be properly determined, because of the low contrast in the images for the three lowest nominal magnifications $\left(M_{0}=5,4\right.$ and 3$)$. For the inverted microscope, the results for $M_{0}=10$ are given in Fig. 6, and for the upright combi-microscope, the results for $M_{0}=25$ are given in Fig. 7; the results for the other magnifications show similar behavior and are therefore not shown. The outcome of the analysis of the measurements does not directly agree with the theoretical values computed by the expressions in (2) and (3) (see solid lines in Figs. 6 and 7). Small corrections on the theoretical estimate for the particle image diameter $d_{\tau}(z)$ are applied for both microscopes. The theoretical value for the particle image diameter for a particle in the object plane $\left(d_{\tau}(0)\right.$, the first two terms in expression (2)) is replaced by the measured particle image diameter at $z=0$. The last term in expression (2) remains unchanged (see dashed lines in Figs. 6 and 


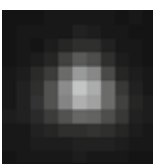

(a)

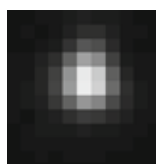

(b)

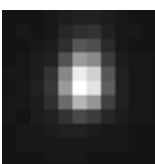

(c)

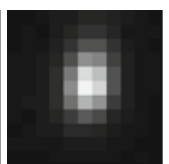

(d)

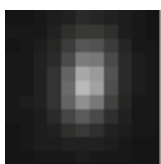

(e)

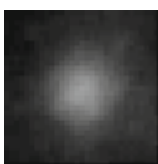

(a)

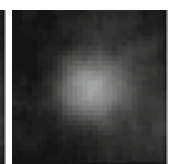

(b)

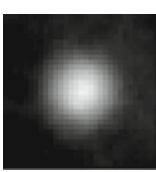

(c)

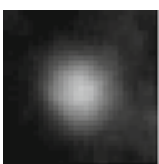

(d)

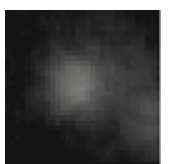

(e)
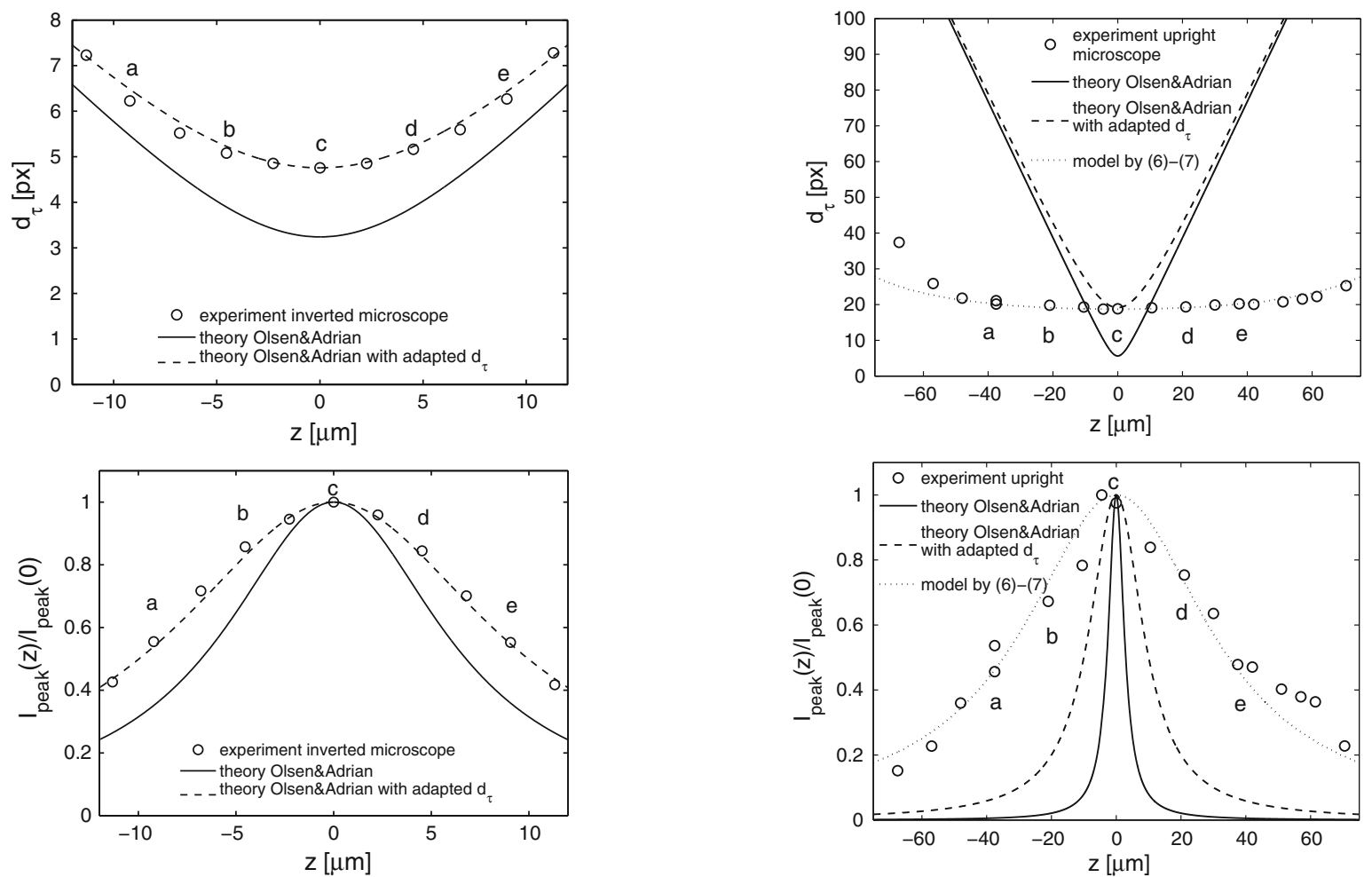

Fig. 6 The measured and calculated particle image diameters and the peak intensities are shown for the inverted microscope with $M_{0}=10$ and NA $=0.3$. When replacing the theoretical value for $d_{\tau}$ by the measured value, the experimental and adapted theoretical values show good agreement. For illustration, particle images are shown for different $z$-positions, indicated by $a-e$

7). This can be seen as incorporating the imperfections of the microscopes into the expression for $d_{\tau}$. Good agreement is shown for both the particle image diameters and the normalized peak intensities for the inverted microscope.

The theoretical values for $d_{\tau}$ are corrected by multiplying them by $1.8 \times, 1.5 \times$, and $2.3 \times$ for the nominal magnifications $M_{0}=20, M_{0}=10$, and $M_{0}=5$ respectively.

For the upright combi-microscope, a correction of the theoretical prediction of the particle image diameter and normalized peak intensity is not sufficient to describe the observed behavior. For this microscope, new empirical models are used to describe their behavior. To describe the particle image diameter:

$d_{\tau}^{2}(z)=\frac{d_{\tau}^{2}(0)}{1-C_{d} z^{2}}$

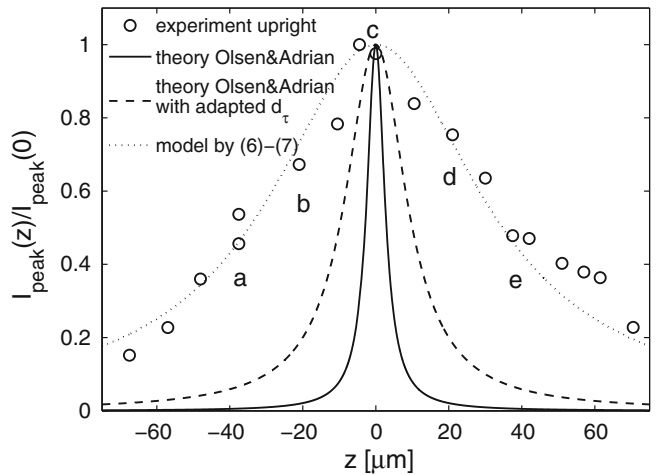

Fig. 7 The measured and calculated particle image diameters and the peak intensities are shown for the upright combi-microscope with $M_{0}=25$ and NA $=0.45$. New models are used to describe the particle image diameter and normalized peak intensities as functions of $z$. For illustration, particle images are shown for different $z$-positions, indicated by $a-e$

and to describe the normalized peak intensity $\tilde{I}_{\text {peak }}$ :

$\tilde{I}_{\text {peak }}=\frac{I(0, z)}{I(0,0)}=\frac{1}{1+C_{i} z^{2}}$

where $C_{d}$ and $C_{i}$ are positive-valued constants. These models are included in Fig. 7.

Severe deviation of the imaging characteristics from the theory can affect the results of the velocity measurements. Since this is the case for the upright combi-microscope, unexpected results for the measured velocity are more likely to occur for this specialized microscope. Moreover, Bourdon et al. (2004b, 2006) have validated the expression for the DOC by inspecting the weighting functions (i.e. the individual contributions to the correlation function). It was shown that the expression holds for a large range of parameters for a microscope which is comparable, 
concerning the complexity, to the inverted microscope used in this study.

\subsection{PIV measurements}

Velocity profiles at the center-plane of the capillary are determined for both microscopes for the different magnifications. The PIV measurements on the upright combimicroscope are repeated with particles with a diameter of $0.56 \mu \mathrm{m}$. The scaling coefficient is determined by dividing the known capillary diameter $(148 \mu \mathrm{m})$ by the diameter (in pixels) of the observed capillary image. Because the Reynolds number of the flow is small $\left(10^{-2}\right)$, the flow can be described as Poiseuille flow with a parabolic flow profile. The measured velocity profiles are compared with the theoretical Hagen-Poiseuille velocity profiles. The only parameter we use to compute the theoretical velocity profiles is the known fixed flow rate of $5 \mu \mathrm{l} / \mathrm{min}$. This value is the prescribed flow rate for the pump and verified in a separate measurement. The fluid, which is pumped through the capillary during one hour, is captured in another syringe, so that no evaporation could occur, and weighted. Together with the measured density of the fluid, the pumped volume can be determined and the flow rate can be verified. The determined flow rate deviated less than $1 \%$ of the prescribed one. All velocities are normalized with the maximum theoretical velocity of the theoretical profiles $(9.7 \mathrm{~mm} / \mathrm{s})$, given the imposed flow rate. The position and distances in the $x$-direction are normalized with the diameter of the capillary $(148 \mu \mathrm{m})$. The direction of the flow velocity does not exactly coincide with the $y$-axis defined

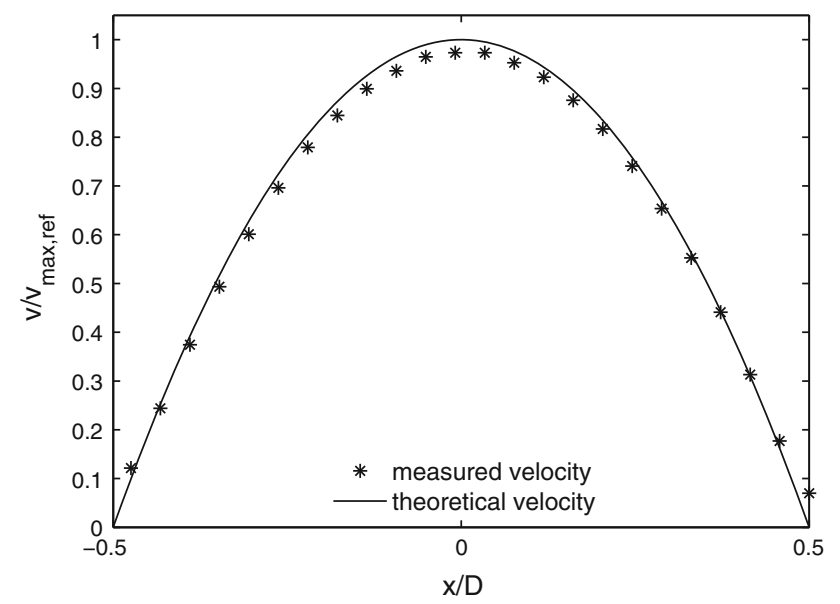

Fig. 8 The measured and the theoretical center-plane velocity profiles are shown for the inverted microscope with $M_{0}=10$ and $\mathrm{NA}=0.3$. The velocities are normalized by using the maximum velocity of the theoretical profile $(9.7 \mathrm{~mm} / \mathrm{s})$, and the distances in the $x$-direction are normalized by using the diameter of the capillary $(148 \mu \mathrm{m})$. For this measurement, the measured maximum velocity is $99 \%$ of the theoretical one

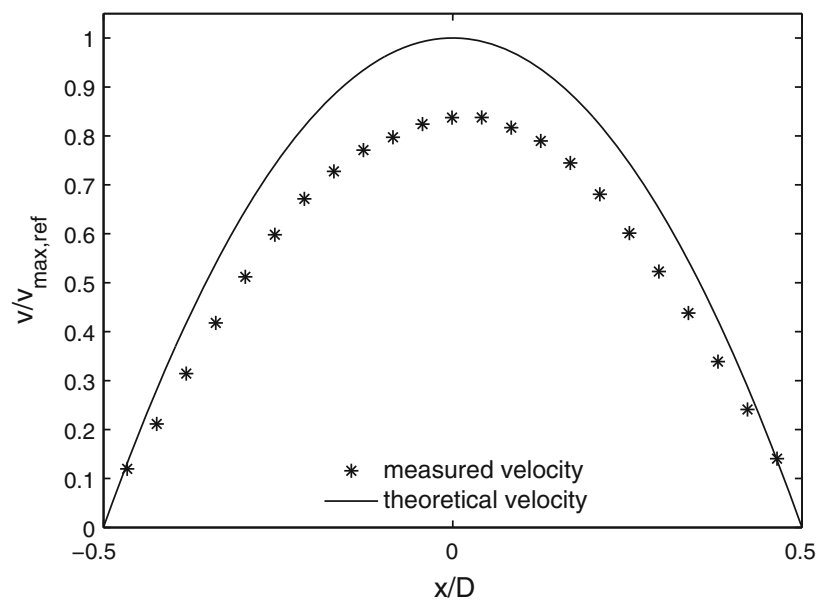

Fig. 9 The measured and theoretical center-plane velocity profiles are shown for the upright combi-microscope with $M_{0}=25$ and NA = 0.45 . The velocities are normalized by using the maximum velocity of the theoretical profile $(9.7 \mathrm{~mm} / \mathrm{s})$, and the distances in the $x$-direction are normalized by using the diameter of the capillary $(148 \mu \mathrm{m})$. For this measurement, the measured maximum velocity is $85 \%$ of the theoretical one

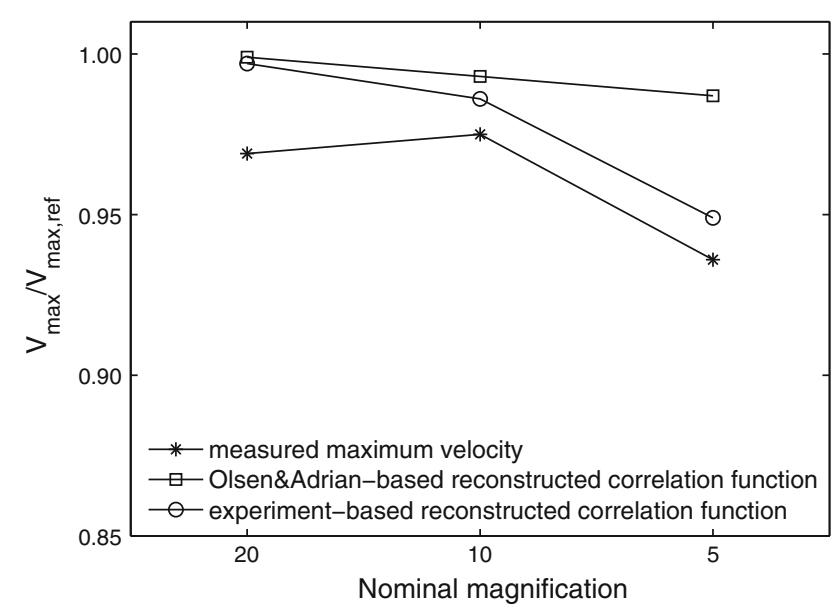

Fig. 10 The measured maximum velocities and the maximum velocities based on the reconstructed correlation functions for the inverted microscope. The reconstructed correlation function is determined for two models for the particle image parameters: Olsen and Adrian-based and experiment-based

for the images. The error in the alignment is estimated to be less than 0.4 degrees. Therefore, the velocity data in subsequent Figs. 8, 9, 10, 11 are velocity magnitudes. In Fig. 8, the measured and theoretical velocity profiles are shown for $M_{0}=10$ obtained with the inverted microscope. The results for the other two magnifications show similar behavior and are therefore not shown. In Fig. 9, the measured and theoretical velocity profiles are shown for $M_{0}=25, \mathrm{NA}=0.45$ resulting from the upright combimicroscope, using $1.28-\mu \mathrm{m}$ particles. The results for other magnifications are similar and are therefore not shown. In 


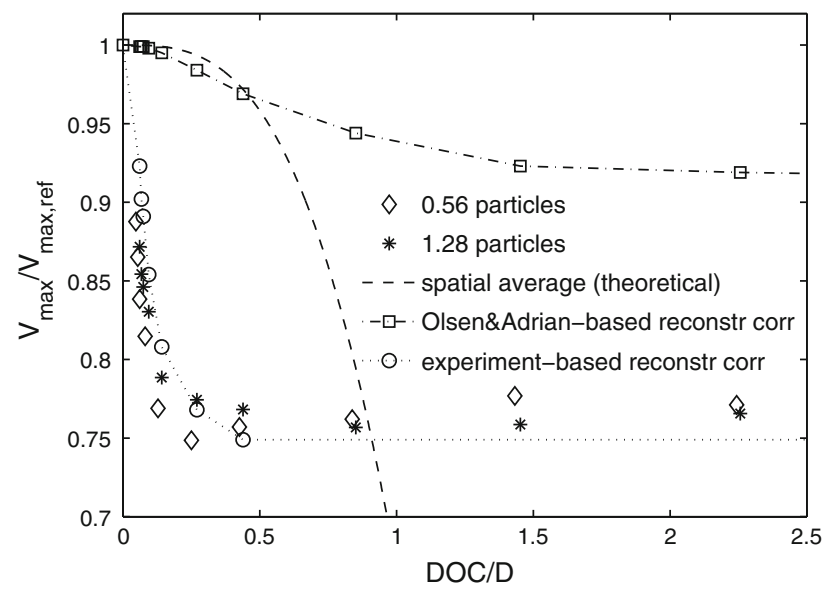

Fig. 11 The measured maximum velocities and the maximum velocities based on the reconstructed correlation functions as a function of the depth-of-correlation relative to the capillary diameter $D$. Data are shown for the upright combi-microscope with $1.28-\mu \mathrm{m}$ diameter tracer particles. Also shown are the measured maximum velocities for the upright combi-microscope with $0.56-\mu \mathrm{m}$-diameter tracer particles

these experiments with the upright combi-microscope, a parabolic profile is found too, but with lower values for the measured velocities.

From the obtained velocity data, the measured maximum velocity is determined by interpolation of a fitted second-order polynomial using the five data points in the center. Using five data points makes the polynomial fit less sensitive for individual measurement errors, and the number of five data points is sufficient to exclude any measurement errors in the data points near the wall of the capillary (Vennemann et al. 2006; Poelma et al. 2008; 2010). Besides, if the DOC influences the measured velocity, it is likely that the averaging effect will be less when $\partial v / \partial x$ is smaller: the velocity differences between the imaged particles within one interrogation window is smaller. From this point of view, any effects due to spatial averaging in the presence of velocity gradients is expected to increase for positions away from the center (Olsen 2009).

The under-estimation of the maximum velocity resulting from depth averaging can now be determined, and this is done for all magnifications that we investigated for both microscopes. These results are shown in Fig. 10 for the inverted microscope and in Fig. 11 for the upright combimicroscope. In Fig. 11, the maximum velocity is plotted as a function of the computed DOC, rather than the magnification, to show the magnitude of the DOC and the relevance of the DOC as a parameter in this study. For both microscopes, the under-estimation of the maximum velocity increases for increasing DOC. The under-estimation of the maximum velocity obtained with the inverted microscope remains small (within 6\%). The under-

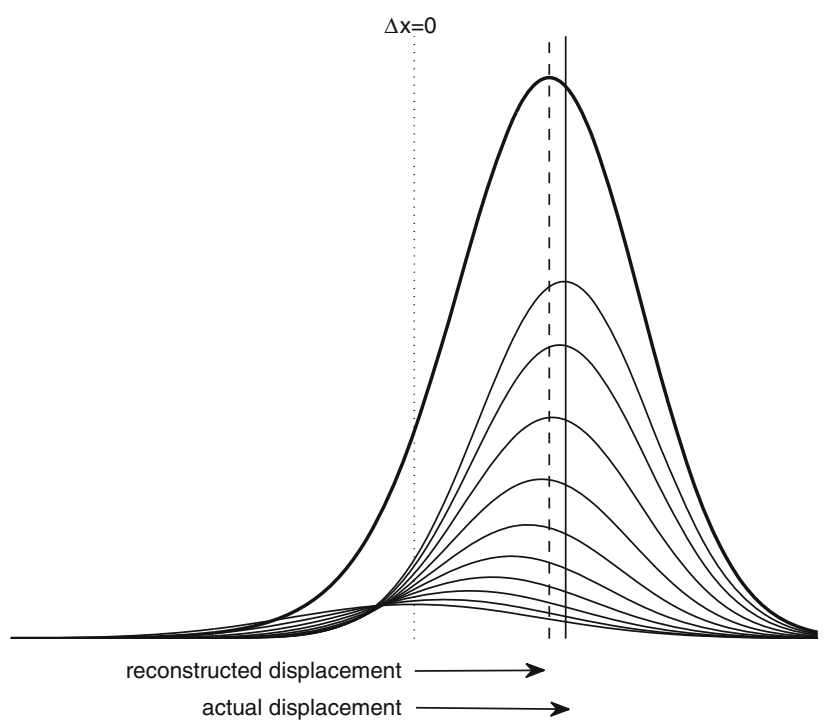

Fig. 12 A reconstructed correlation function is shown (thick line) together with the individual contributing correlation functions (thin lines). Only a limited number of individual contributing correlation functions is shown for illustration. The location of the maximum of the correlation function (dashed line) does not coincide with the location of the maximum of the displacement (solid line). The height of the reconstructed correlation function is reduced in order to show the reconstructed and individual correlation functions in one figure

estimation of the maximum velocity obtained with the upright combi-microscope becomes considerably larger for large DOC: up to $25 \%$. In both figures, also the expected maximum velocities resulting from the reconstructed correlation functions are shown. Figure 12 shows an example of a reconstructed correlation function. Two models are used to describe the particle image parameters involved in the reconstructed correlation function: (a) the theoretical particle image diameters and normalized peak intensities calculated by (2) and (3) (Olsen and Adrian-based reconstructed correlation function), and (b) the measured particle image diameters and normalized peak intensities from the first part of this study (experiment-based reconstructed correlation function). The measured particle image parameters obtained from the inverted microscope can be described by the theoretical estimates, given by (2)-(3), with a small correction. The measured particle image parameters obtained from the upright combi-microscope can be described by the model in (6)-(7) ; see Sect. 3.1 In addition, the spatial average is also given in Fig. 11, which is the average velocity in the DOC: $\frac{1}{D O C} \int_{-\mathrm{DOC} / 2}^{\mathrm{DOC} / 2} v(z) \mathrm{d} z$. For the models, the $1.28-\mu \mathrm{m}$ particles are used. Only the measured maximum velocities are shown for the $0.56-\mu \mathrm{m}$ particles. Based on these measured maximum velocities, no exceptional deviations are expected due to different particle sizes and no additional measurements and analysis are done for the $0.56-\mu \mathrm{m}$ particles. 
The measured maximum velocities resulting from the inverted microscope are well predicted by using reconstructed correlation functions with both models. The maximum error occurring in the measured maximum velocity is $6 \%$. The deviation of the measured maximum velocity from the expected maximum velocity for $M_{0}=20$ is probably due to the inaccuracy made in determining the scaling coefficient defined by the ratio of the capillary diameter $(148 \mu \mathrm{m})$ and the diameter (in pixels) of the observed capillary image. Dividing the scaling coefficients corresponding to the cases $M_{0}=10$ and $M_{0}=5$ by two and four, respectively, the under-estimation of the expected maximum velocity by the measured maximum velocity is less than $1 \%$.

The under-estimation of the maximum velocity when using the measured maximum velocity is for the upright combi-microscope much larger than for the inverted microscope. From Fig. 11, it is clear that the measured velocity does not match the theoretical spatial average (shown as the dashed line). A reconstructed correlation function based on the theory of Olsen and Adrian given by equations (2)-(3) predicts a value for the maximum velocity that is also not correctly representing the measured velocity. But the under-estimation can be approximated well when a reconstructed correlation function is used that is based on the measured values for the particle image diameter and the intensity distribution for all the magnifications in the tested range (see Fig. 11). Since the underestimation is known, a correction of the measured maximum velocity is possible, and derived flow parameters, such as the flow rate, can be obtained.

\section{Discussion}

The DOC gives an indication of the depth over which the particles contribute to the correlation function. If the DOC is sufficiently small, the measured velocity is a good approximation of the actual velocity when gradients are present. But when the DOC increases, the way the measured velocity is affected is not straightforward. Because a large DOC cannot always be avoided (especially when a microscope objective with a low magnification and low numerical aperture is used, but also when large particles are used), it is useful to know how the measured and actual flow velocities are related. Moreover, the computed value for the DOC is not always in agreement with the actual behavior, as is demonstrated in this paper. The way how particles are imaged plays a crucial role: if this deviates far from the theory, a closer look on this is required. Although one may find that for most microscopes the optical properties are described well by established descriptions, it is important to validate this against actual measurements. A good starting point is to compare the theoretical value and the measured value of the particle image diameter for a particle in focus. Significant difference between the predicted and measured particle image diameter can be seen as a strong indication for further investigation. This is especially the case for specialized microscopes, as is the case for the combi-microscope described in this paper. To obtain the correct velocities, a procedure is recommended which is generally applicable. But for inverted microscopes, such as the one presented in this study, small improvements can be expected, and applying the existing theory for the DOC will probably suffice.

A recommended procedure is as follows:

1. Perform a measurement to determine how the particles are imaged by a given microscope as a function of the distance from the focal plane and determine the characteristic parameters necessary to describe the diameter, maximum intensity and shape of the particle images for different $z$-positions.

2. Find a generalized function for the characteristic parameters that fits the measured optical characteristics as a function of $z .^{4}$

3. Determine the individual contributions of particle images to the correlation function as a function of $z$, i.e. the individual correlation functions at each $z$ position. An individual correlation function can be obtained by computing the auto-correlation of the particle image and centering this around the corresponding displacement.

4. Then, reconstruct a correlation function by summing up all individual correlation functions from step 3 .

5. Determine the peak location in the reconstructed correlation function. The measured displacement relative to the actual displacement in the nominal focal plane gives the desired correction for the actual velocity, based on the reconstructed correlation function.

The last few steps are obviously only possible if the (approximate) shape of the velocity profile is known. Fortunately, this is often the case in microfluidics (low Reynolds number flow through channels, capillaries or blood vessels). For more complex flows, one needs to limit the DOC to small values (and check this, see step 1) to avoid complex spatial-averaging effects.

\footnotetext{
${ }^{4}$ One could also consider to adapt physical parameters (e.g. the numerical aperture) to see if this results in a correct description of the diameter, maximum intensity and shape of the particle images for different $z$-positions. In this study, only adapting physical parameters was not sufficient.
} 


\section{Conclusions}

In micro-PIV, the DOC is an important experimental parameter. For most microscopes objectives, the numerical aperture NA is proportional to the magnification, i.e. objectives with low magnification also tend to have a lower NA in comparison with objectives with a higher magnification. At the same time, the DOC is inversely proportional to the NA. In practice, this means that for micro-PIV measurements with a large field-of-view, i.e. low magnification, the NA is small, and the DOC is large. To achieve a very small DOC, implies using a large NA and high magnification, which implies a small field-of-view. With increasing DOC, the contribution of the out-of-focus particles becomes more important. This becomes an issue in the presence of significant out-of-plane gradients of the displacement (i.e., velocity). In this paper, it is shown that this can lead to an under-estimation of the measured displacement. In the case of Poiseuille flow through a round capillary, this can lead to a significant under-estimation of the maximum velocity in the flow up to $25 \%$. When the under-estimation of the maximum velocity can be quantified, the exact velocity profile and resulting flow rate can be determined, assuming a parabolic velocity profile in the case of a Poiseuille flow. For two types of microscopes, i.e. a conventional inverted microscope and a specialized combined upright stereoscopic/single-view microscope, a study is done on whether a correction on the measured velocity is needed such that the exact velocity profile and resulting flow rate can be determined. For this purpose, PIV measurements were done in a round capillary with a diameter of $148 \mu \mathrm{m}$. Both types of microscopes give different results, owing to the different imaging characteristics of these microscopes. To understand the outcome better, a closer look is taken on the imaging of the particles by the microscopes. While the present inverted microscope, which presumably approximates the single thin lens closer, gives results that are in reasonable agreement with theoretical descriptions by Olsen and Adrian (2000), the combined microscope has a very different behavior.

For the inverted microscope, the maximum velocities in the capillary are well predicted (within 6\%), even at low magnification, i.e. large DOC. The way particles are imaged by the inverted microscope matches the theory sufficiently. This can be slightly improved when in the theoretical expression for the particle image diameter, only the value for the particle image diameter in the focal plane is substituted by the measured value for the particle image diameter in the focal plane.

But when looking at the particle images created by the upright combi-microscope, the theory of Olsen and Adrian does not adequately describe the shape and size of the particle images as a function of the out-of-plane distance.
Over a much longer $z$-range their increase in diameter and decrease in peak intensity is minimal for particles going away from the focal plane than can be expected by the theory. This behavior has consequences for the outcome of the PIV analysis. Since the contribution of the particles with lower velocities to the correlation function becomes larger, the maximum of the correlation function will be shifted toward lower values for the displacement. To predict the underestimation, the spatial average is not a good measure for the measured maximum velocity, even not for low magnifications where the DOC exceeds the diameter of the capillary. A better estimation of the measured maximum velocity can be achieved when the displacement is determined by a reconstructed correlation function based on experimentally defined particle image parameters. For the upright combimicroscope used in this study, the measured maximum velocities for magnifications smaller than $10 \times$ remain unchanged and equal to $75 \%$ of the theoretical value. Based on this fact, the real velocity profile and the flow rate can be derived from the measured values.

Acknowledgments The authors would like to thank Professor Carl Meinhart (UCSB) for his valuable feedback in the early stages of this study and the two anonymous reviewers for their useful remarks and suggestions. This work is financially supported by the Medical Delta initiative.

Open Access This article is distributed under the terms of the Creative Commons Attribution Noncommercial License which permits any noncommercial use, distribution, and reproduction in any medium, provided the original author(s) and source are credited.

\section{References}

Adrian RJ, Westerweel J (2010) Particle image velocimetry. Cambridge University Press, Cambridge (in press)

Adrian RJ, Yao CS (1985) Pulsed laser technique application to liquid and gaseous flows and the scattering power of seed materials. Appl Optics 24(1):42-52

Bourdon CJ, Olsen MG, Gorby AD (2004a) Power-filter technique for modifying depth of correlation in micro-PIV experiments. Exp Fluids 37(2):263-271

Bourdon CJ, Olsen MG, Gorby AD (2004b) Validation of an analytical solution for depth of correlation in microscopic particle image velocimetry. Meas Sci Technol 15(2):318-327

Bourdon CJ, Olsen MG, Gorby AD (2006) The depth of correlation in micro-PIV for high numerical aperture and immersion objectives. J Fluids Eng 128(4):883-886

Fouras A, Dusting J, Lewis R, Hourigan K (2007) Three-dimensional synchrotron $\mathrm{X}$-ray particle image velocimetry. J Appl Phys 102(6):064,916

Fouras A, Jacono DL, Nguyen CV, Hourigan K (2009) Volumetric correlation PIV: a new technique for $3 \mathrm{~d}$ velocity vector field measurement. Exp Fluids 47(4-5):569-577

Lee JY, Ji HS, Lee SJ (2007) Micro-PIV measurements of blood flow in extraembryonic blood vessels of chicken embryos. Physiol Meas 28(10):1149-1162 
Meinhart CD, Wereley ST (2003) The theory of diffraction-limited resolution in microparticle image velocimetry. Meas Sci Technol 14(7):1047-1053

Meinhart CD, Wereley ST, Santiago JG (2000) A PIV algorithm for estimating time-averaged velocity fields. J Fluids Eng 122(2):285-289

Mielnik MM, Saetran LR (2006) Selective seeding for micro-PIV. Exp Fluids 41(2):155-159

Olsen MG (2009) Directional dependence of depth of correlation due to in-plane fluid shear in microscopic particle image velocimetry. Meas Sci Technol 20(1):015,402

Olsen MG, Adrian RJ (2000) Out-of-focus effects on particle image visibility and correlation in microscopic particle image velocimetry. Exp Fluids 29(7):S166-S174

Poelma C, Vennemann P, Lindken R, Westerweel J (2008) In vivo blood flow and wall shear stress measurements in the vitelline network. Exp Fluids 45(4):703-713

Poelma C, Van der Heiden K, Hierck BP, Poelmann RE, Westerweel J (2010) Measurements of the wall shear stress distribution in the outflow tract of an embryonic chicken heart. J R Soc Interface 7(42):91-103

Rossi M, Cierpka C, Segura R, Kähler CJ (2010) On the effect of particle image intensity and image preprocessing on depth of correlation in micro-PIV. In: 15th International symposium on applications of laser techniques to fluid mechanics, Lisbon, Portugal, 05-08 July, 2010

Sugii Y, Nishio S, Okamoto K (2002) In vivo PIV measurement of red blood cell velocity field in microvessels considering mesentery motion. Physiol Meas 23(2):403-416

Vennemann P, Kiger K, Lindken R, Groenendijk B, Stekelenburg-de Vos S, Ten Hagen T, Ursem N, Poelmann R, Westerweel J, Hierck BP (2006) In vivo micro particle image velocimetry measurements of blood-plasma in the embryonic avian heart. J Biomech 39(7):1191-1200

White FM (2008) Fluid mechanics. 6th edn. McGraw-Hill, New York 